\title{
Explore the Innovation Path of College Students' Internet Public Opinion Response Based on the Theory of Affective Thinking
}

\author{
Sun Lanxin \\ Guangxi Polytechnic of Construction, Guangxi, Nanning, 530007, China \\ sunlanxin@126.com
}

Keywords: Emotional thinking; Internet public opinion; monitoring; guidance; college students.

\begin{abstract}
The virtual environment formed under the media has a profound impact on college students' thinking and behavior, and the network public opinion is bred. Starting with the characteristics of emotional thinking function, accomplishment, cognition and college students' network public opinion, this paper innovates the monitoring and guiding path of college students' network public opinion, puts forward the establishment of correct moral outlook, outlook on life, world outlook and values, constructs the analysis system of college students' network public opinion, and establishes the monitoring and guidance mechanism of linkage response.
\end{abstract}

\section{Emotional thinking and college students' Internet public opinion.}

Emotional thinking, different from emotion, is a combination of perceptual subjective experience and rational logical form to guide people's psychological and behavioral activities. With the advent of the media age, Internet public opinion has been born. With the network as the carrier and the event as the core, the University students' network public opinion formed by the expression, dissemination and interaction of emotions, attitudes, opinions and opinions has come into people's view. Understanding the function of emotional thinking and the characteristics of University Students' network public opinion will help to innovate the response of University Students' network public opinion. The path is very necessary.

\subsection{The function of emotional thinking.}

Emotional thinking takes "emotion" as the core of logical thinking activities, through logical thinking, rational restraint or promotion of varying degrees of emotional experience in the cognitive activities of the effectiveness of non-intellectual emotional factors to play an active role. It has the functions of power, regulation and guidance, and permeates all aspects of human social life. The power function of emotional thinking refers to the effective force that drives people's behavior to move forward or backward. In practice, the subject will evaluate the information of the object according to its own needs in accordance with its own interests. Pleasure, positive emotional experience and logical thinking will produce a strong driving force to promote people to better explore the law and understand the truth; vice versa. The regulating function of emotional thinking has the ability to organize or disrupt students' activities. It requires the subject to regulate and control his own behavior effectively through positive emotion and quick and clear thinking, to overcome the interference factors encountered in the activities, and to ensure the orderly and efficient operation of the activities. At the same time, the function of emotional thinking cannot be ignored, refers to the effective enlightenment and solution of contradictions between themselves and others, so as to make it unimpeded and achieve social harmony and order. That is, "through the emotional dredging to eliminate the individual's psychological resistance to the input of external information, in order to be reasonable and reasonable." [1] realizes friendly interpersonal relationship and promotes harmony between people. 


\subsection{The characteristics of College Students' Internet public opinion.}

College students' network public opinion is a collection of related opinions, opinions, emotions and attitudes expressed by relying on the media and taking college students as the object of discussion on some public affairs, current affairs hot spots or social focus issues. It has the characteristics of subjective initiative, emotional sensibility and multi-dimensional value. It expresses its own consciousness judgment, emotional cognition and value orientation of an event through the computer internet. Subjective initiative refers to the University Students' comments, knowledge and feelings about a public event on the Internet, with their own subjective judgment. This judgment can be positive or negative. Emotional perceptualization, as its name implies, means that college students' personal emotions are subject to subjective fluctuations and uncertainties, influenced by the environment, personal experience and other factors, and their behavior and speech are easy to change. The multi-dimension of value refers to the complexity and diversity of College students' network public opinion. With the development and change of social politics, economy and culture, the contradiction and conflict of social values are highlighted. The multi-angle cultural perspective, the pluralistic value of interests, and the multi-faceted criteria of evaluation all have an impact on College Students' own values, ideals and beliefs. Make it through the network of a public event, focus on issues related to the expression of views, opinions have a valuable multi-dimensional characteristics.

College students reflect their feelings and thoughts through the network public opinion. In order to enable students to use the network correctly and guide students to have objective and rational, logical judgment to develop network public opinion, it is necessary to give full play to the positive function of emotional thinking, standardize college students' network behavior, and realize the supervision and guidance of College Students' network public opinion.

\section{The necessity of standardizing college students' Internet public opinion based on emotional thinking.}

The purpose of emotional thinking is to create a meaning world, meaning space and give the world a new meaning, which originates from real life and is higher than real life and conforms to the law of beauty. [2] It can regulate and supervise college students' network public opinion through the fresh experience of emotion and the rational control of thinking.

\subsection{Cultivating effective personality cultivation and weakening trust crisis through effective use of emotional thinking.}

The development of network technology provides a more convenient way to present social phenomena and citizens' wishes. College students express their views, opinions and attitudes through the Internet. However, due to the lack of rational analysis by some college students, they express negative rebellious opinions against the emergence of social phenomena or the exposure of illegal and criminal news by the media. They doubt society, others and trigger a crisis of social trust, which leads to the decline of collective cohesion and centripetal force. A weak sense of integrity, breeding undeserved treason and endangering campus safety. We must give full play to the positive incentive effect of emotional thinking, consciously form a good personality, "no good and no evil is the body of the mind, there is good and evil is the action of intention, knowing good and knowing evil is conscience, for good and evil is the latter." [3] Explain that moral character plays a great role in guiding people's behavior activities, urge students to form the behavior habit of honesty and trustworthiness, restrain their network activities subtly, make communication more trust, less doubt, weaken the crisis of trust in the current society, and promote the harmony, health and order of the network society. Stable development.

\subsection{Through the positive guidance of emotional thinking, we should establish correct value orientation and reduce group conflicts.}

Scheler once pointed out that "everything that we recognize as morally important in a person or group must be, always indirectly, reduced to the special structure of the potential of love and hate for 
the actions we love and hate, to the order of love that governs them and manifests itself in all emotional impulses." [4] It refers to the order in which great emotional power is restricted by reason and has applicability, which is the effect of emotional thinking. In the network virtual environment, through positive emotional thinking to seek college students' emotional identity, establish the core values of Communist beliefs, guide students to conduct rational network public opinion activities. Taking the socialist core values as its orientation, we should infiltrate students' ideology, strengthen their Marxist beliefs and socialist beliefs, adhere to the leadership of the Communist Party of China, establish the value orientation of serving the people, create an optimistic atmosphere of public opinion, so that students can distinguish right from wrong rationally, be sensible and be irresponsible. What temptation to make a socialist value orientation of choice, resolve contradictions, reduce group conflicts, create a positive, harmonious and orderly network of public opinion environment.

\subsection{Through the rational norms of emotional thinking, we should control misleading public opinion anomie and maintain campus stability.}

Through the network students can express their ideas and wishes, but because of the universality, convenience and rapidity of the network, the network public opinion has become a loudspeaker for some lawless elements to make false news and rumors and false statements, leading to false information and public opinion anomie, misleading students' behavior and ideas, utilitarianism and individualism flourishing. OK, no sense of collective and social responsibility, create and disseminate false information, making public opinion loss of orderly norms. Chaotic network situation and disorderly norms lead to the imbalance of College students' network communication and information transmission, which is easy to mislead College students, and even lead to group unrest and disturbance. Therefore, it is necessary to give full play to the positive regulatory function of emotional thinking, rationally control the unhealthy emotions of College students, overcome the interference of various erroneous remarks, and create an orderly network environment, so that all kinds of network public opinion can truly reflect students' attitudes and opinions on various school events, and can also make suggestions on the construction, development and service of schools. Put forward reasonable opinions and suggestions, improve the school rules and regulations implementation and administrative management transparency, reduce campus emergencies, and effectively maintain and achieve campus harmony and stability.

\section{Innovative ways to monitor and guide college students' Internet public opinion.}

With the rapid development of information society, the importance of emotional thinking in the choice of tool rationality and value rationality has been highlighted. Emotional thinking has been used to innovate the monitoring and guiding path of network public opinion, so as to make healthy and upward network public opinion become a driving force for the development of schools and ensure the harmony and stability of schools.

\subsection{Giving full play to the function of emotional thinking and establishing a correct moral outlook, world outlook, outlook on life and values.}

Through the motive force, regulation and guidance function of emotional thinking, college students should be reasonably guided to correctly analyze and judge the content of network information, and to give full play to their emotional enormous efficacy in conducting network commentary and attention activities around political issues, ideological viewpoints, social trends, etc., so as to rationally establish patriotism and collective ownership. The emotional values of righteousness follow the order of the network and overcome the harm of bad information. Under the positive effect of emotional thinking, students should firmly establish the ideal and belief of building socialist modernization and correct moral outlook, world outlook, outlook on life and values.

In modern information society, college students' network communication is becoming more and more frequent and diversified. Effective monitoring and guidance of network public opinion has become an important part of campus management. College students can be nurtured by campus 
culture, teachers' teaching, students' friendship to wash away the soul turbidity, forming a positive emotional thinking, rational adjustment of self-psychological state and behavior. In the network activities, we can grasp the sense of discretion, seek common ground while reserving differences, achieve harmonious coexistence with other people's emotional needs and value judgments in the expression of our own views and judgments, distinguish between right and wrong rationally, make our own wishes and purposes come true through reasonable network words and deeds, and control our own improper emotions through thinking. In order to create a rich spiritual home, we should rationally recognize the various kinds of information conveyed by the network actively resolve contradictions when conducting public opinion, reduce confrontation. Give play to the coordinating role of emotional mechanism, thinking mechanism and physiological mechanism in college students' network behavior, make students' network public opinion full of positive energy, better promote the harmonious and orderly development between students and students, students and schools. The formation of outlook on life, values, world outlook and moral outlook depends on nurturing students with healthy and noble sentiments and rational thinking guidance. It can strengthen the construction of campus culture, make students have beautiful feelings and temperament under the influence of beautiful environment, and then consciously carry out network public opinion and network practice. Using network morality to restrain one's own network behavior, whether on the Internet or in real life, one can be strict with oneself, abide by various rules, constantly cultivate one's own moral and spiritual realm, form a correct world outlook, outlook on life, values, and promote the harmonious development of the campus.

\subsection{Cultivating emotional thinking literacy and constructing a network public opinion analysis system for college students.}

Focus on the cultivation of emotional thinking literacy, cultivate students' emotional awareness, love consciousness, strong will, firm belief as a midwife to deal with college students' network public opinion, to achieve a coordinated, healthy and sustainable development of the network environment. The accomplishment of emotional thinking includes cognition, affection, will and belief. Through the correct cognition of network public opinion, analyzing relevant network information, penetrating the students' own emotional morality, combining their own moral will and indestructible ideals and beliefs, the network public opinion analysis system is constructed, that is, the collection channel is established. Tao, strengthen the predictive power, improve the intervention mechanism, guide college students rational use of the network, adhere to the combination of self-discipline and heteronomy, make reasonable judgments and choices on the input and output of information and public opinion, to achieve efficient supervision and management of College Students' network public opinion.

College students should cultivate themselves with emotion, control their behavior with intelligence, consciously maintain the norms of network society and develop network public opinion. In practice, we should transform the psychological needs of informed consent into the unremitting pursuit of truth, goodness and beauty. First of all, through rational cognition, we should strictly check the legitimacy of the network information sources, achieve the norms of information release, require specialized personnel, attach great importance to the management of the source of network information in cognition, eliminate bad information release, so that public opinion supervision from the source to ensure reasonable. Secondly, through rational thinking to achieve effective prediction and correct judgement of public opinion. Any subjective comment has individual emotional experience, needs rational thinking to regulate control, requires schools to carry out rational monitoring of network public opinion, improve the monitoring network system, make a rational prediction of the development trend of events, cannot be emotional, the network public opinion caused by the emergencies of early warning, to avoid The occurrence of group incidents. Finally, the establishment and improvement of the intervention mechanism cannot be separated from the moral will power of emotional thinking and the promotion of rational beliefs. Through the moral restraint and emotional incentive function of emotional thinking, the intervention mechanism of multi-party participation and coping with both on-line and off-line should be established to reasonably interfere 
with students' unhealthy network public opinion behavior, and through the responsibility implementation system, various interactive strategies and behavior plans suitable for students' actual network public opinion should be established to promote students' behavior. Students can improve their emotional thinking ability, self-discipline and restrain their own online speech, logical input and output of information in line with the objective and practical judgment. Only in this way can we prevent in the future, curb the adverse consequences, and reduce the possible mass incidents caused by college students 'network public opinion.

\subsection{Strengthen the cognition of emotional thinking and create the "123" linkage mechanism of monitoring and guidance.}

The supervision and guidance of College students' network public opinion cannot be separated from the establishment of university network management mechanism, strengthen the managers' cognitive ability of emotional thinking, and create a "one-two-three" linkage mechanism: a core - to establish a management team of college public opinion, giving full play to the rational cognitive function of emotional thinking, rational and full of emotional functions. The working group can be composed of school psychological consultation center, propaganda information department, logistic security and other personnel, to have a rich emotional life experience, but also must have a rational and objective analysis ability, can take the initiative to effectively integrate a large number of scattered isolated network information, forward-looking and predictive network. Public opinion events to prevent. At the same time, collecting and releasing information timely, accurately and comprehensively, starting from the emotional experience, we can quickly realize the spiritual resonance with college students, teach students with positive and reasonable moral principles, quickly and humanely respond to the focus of attention of student groups, stabilize students' emotions, standardize the network public opinion environment, and maintain the school. Park is stable and United.

Two teams - to create a network of information technology monitoring team and network ideological and political education team, to emotional people, wise practice, reasonable norms of College Students' network public opinion. Network information technology monitoring team is mainly composed of experts and managers who are familiar with network technology. They always test the network security system and supervise the information transmitted by the network rationally and strictly. Public opinion informants must analyze, summarize and judge all kinds of information on the network rationally and clearly while possessing rich emotions. We should grasp the changes of students' ideological and emotional development, grasp the dynamic of public opinion according to experience and logical analysis, predict the occurrence and development of events and possible crises timely and accurately, and deal with the related adverse network public opinion immediately. At the same time, the ideological quality of college students has a tremendous impact on the network public opinion. Establishing a network ideological and political education team, which is composed of professional teachers, political theory teachers and counselors, can produce effect on students' network public opinion emotionally and in action, and prevent the emergence of bad public opinion from the source. Network ideological and political education can include various moving deeds, soul chicken soup position, red patriotism base education and other columns, using emotional thinking in the daily life of students to learn, positive and optimistic treatment of related hot spots, focus issues, guide students to establish honesty and trustworthiness, equality and mutual assistance, self-improvement ideology. Reasonably analyze the right and wrong of network information, true and false, good and bad, and earnestly maintain the orderliness of campus network space.

Three systems - from the influence of the development of emotional thinking, construct the network moral education system, network behavior norms system, network emotional cognition system, so that college students consciously restrain network public opinion activities from the ideological consciousness. Emotional thinking can influence and educate students' ethics and morality. While giving full play to emotional impetus, we should control our own behavior rationally, strengthen moral consciousness and legal concept, set up correct network ethics, consciously abide by network ethics standards, and use network resources civilly. Furthermore, the formulation and 
implementation of the network behavior norm system requires emotional thinking to play a rational cognitive function, so that college students from the habit of behavior to develop, consciously and rationally resist the pollution of harmful information on the network, targeted network public opinion activities, comply with the network management system, prevent deceptive bad information. Intrusion. Finally, the establishment of network emotional cognition system should be based on the judgment, flow and choice of emotion. It is through the guidance of this theory that network public opinion can realize the monitoring and management of College Students' public opinion. Emotion judgment is the affirmation or negation of a certain kind of emotion by college students in network activities. The feeling of increasing force can promote the correctness of College Students' behavior, otherwise, it is not. Emotional flow refers to the emotional changes with the occurrence and development of events, and the cognitive content will also change. Emotional choice is related to the role of College Students' inherent knowledge cognition in choosing emotions, affecting the formation of College Students' network emotions. The establishment of network emotional cognition system is to regulate the role of College Students' emotions reasonably through relevant rational cognition, and overcome the role of pessimism, jealousy, hatred and other negative emotional factors. We can standardize college students 'network public opinion by establishing affective choice system, affective restraint system and affective education system, achieve the combination of campus network sentiment and reason, avoid disorderly network behavior caused by improper emotions, and realize the supervision and guidance of network public opinion.

\section{Acknowledgement}

Foundation Project: Guangxi University Ideological and Political Education Theory and Practice Project in 2014: Self-media-based monitoring and guidance of College students' network public opinion path research (No. 2014 LSZ081).

\section{References}

[1] LI Liao. Relationship between empathy and prosocial behavior among adolescents [J]. Psychological Journal, 1990, (1): 72-77.

[2] Chen Zhongli, et al. Thinking style and social development [M]. Beijing: Social Sciences Literature Press, 2001:210.

[3] [Ming] Wang Yangming. Organization and compilation of the Chinese Confucius Society. Selected from the book of Yangming Quanshu in Biography. Beijing: Higher Education Press, 2004:28-29.

[4] Selected byLiu Xiaofeng. Scheler's Anthology [M]. Shanghai: Sanlian bookstore, 1999:741-740. 